\title{
Catatonic Disorder due to Wilson's disease - A rare presentation
}

\author{
Tanushree Bhattacharya ${ }^{1}$, Asish Debnath ${ }^{2}$ and Sharmila Sarkar ${ }^{3}$ \\ ${ }^{1}$ Department of Psychiatry, Calcutta National Medical College \& Hospital, Kolkata, India. \\ 2,3 Department of Psychiatry, Calcutta National Medical College \& Hospital (2nd campus), Kolkata, India \\ Email : tanushree19april@gmail.com
}

\section{ABSTRACT}

Wilson disease (WD) is a relatively rare autosomal recessive disorder caused by the mutation of ATP7B gene, resulting in impaired transportation of copper in the body which is then deposited in various organs such as liver, brain and kidney. Catatonia at first presentation in WD has rarely been reported. Here we report a case of a 14 year old boy who presented with catatonia among other neuropsychiatric features and who was later diagnosed with Wilson's disease. He responded well to treatment with Copper chelators, olanzapine and lorazepam. Though uncommon, a diagnosis of Wilson's disease should be considered in the evaluation of adolescents and young adults presenting with psychiatric manifestations \&/or neurological features.

Key words : Treatment, Wilson's Disease, Catatonia.

\section{INTRODUCTION}

Normal dietary consumption and absorption of copper exceedthe metabolic need, and homeostasis of this element is maintainedexclusively by the biliary excretion of copper. Wilson's disease (WD) is an autosomal recessively inherited disorder in which defective biliary excretionof copper occursdue to mutations of the ATP7B gene on chromosome $13^{[1,2]}$, which encodes a copper-transportingP-type ATPase (ATP7B), hence leading to copper accumulation, particularly in liver and brain ${ }^{[3,4]}$. Incidence may be as high as 1 in 30,000 andclinical presentation can vary widely ${ }^{[5]}$. The most common presentations are with liver disease or neuropsychiatric disturbances. Asymptomatic patients are most oftendetected by family screening. Lifetime prevalence of psychiatric disorders in patients with WD is estimated to range between $30 \%$ and $100 \%{ }^{[3,6,7]}$. The psychiatric manifestations of $\mathrm{WD}$ can be categorized into: personality changes, affective disorders, psychosis, cognitive impairment, and others. Personality change, affective disorders including depression, and cognitive impairment are common, and schizophrenia-like psychoses are rare ${ }^{[6,7]}$. The lifetime prevalence of persistent personality change ranges between $46-71 \%{ }^{[8,9]}$. Schizophreniform disorders, catatonia, and hallucinations are no more common in WD than in the general population ${ }^{[3,7]}$ but psychosis and catatonia occurred somewhat more commonly (8\% each) in neurological $\mathrm{WD}^{[9]}$. In children with Wilson's disease, declining schoolperformance, impulsiveness, labile mood, sexual exhibitionism, and inappropriate behaviour are observed ${ }^{[10]}$. The 
clinical hallmark of Wilson's disease is the KayserFleischerring, which is present in $95 \%$ of patients with neurologicsymptoms and somewhat over half of those without neurologic symptoms ${ }^{[11,12]}$. In children presenting with liver disease, Kayser-Fleischer rings are usually absent ${ }^{[13]}$. Kayser-Fleischer rings are caused by deposition of copper in Descemet's membraneof the cornea. A slit-lamp examination by an experiencedobserver is required to identify Kayser-Fleischer rings ${ }^{[14]}$.

Clinical improvement in WD with treatment is generally limited to the first five years of symptoms and the first two years of WD treatment ${ }^{[6,15]}$. Untreated Wilson's disease is universally fatal, with most patients dying from liver disease and a minority from complications of progressive neurologic disease. With chelation treatment and liver transplantation, prolonged survival has become the norm ${ }^{[16,17,18]}$. Hence early detection and treatment of WD is mandatory for a good outcome. Treatments include cupriuretic copper chelators (penicillamine and trientine). Dimercaprol a chelator was the earliest treatment for WD. Controlled trials of psychotropic treatment, psychotherapy, and family therapy have not been carried out in $\mathrm{WD}^{[19]}$. Anecdote suggests that patients with WD are predisposed to extrapy ramidalside-effects with antipsychotics ${ }^{[20]}$. Electroconvulsive therapy (ECT) for psychoticde pression in a patient with WD led to a 3-min motor seizure followed by a switch to mania ${ }^{[21]}$. However, there are a few case reports of successful ECT treatment for psychiatric symptoms in $\mathrm{WD}^{[22,23]}$.

\section{CASE REPORT}

A 14 year old Muslim boy from a rural background was brought by his mother to the Psychiatry OPD, being referred by a general physician (RMP) with complaints of insidious onset of childish behaviour and forgetting of all previously learned skills since last 1 year, stiffness of upper limbs and diminished communication since last 8 months and indifference to surroundings along with occasional agitation since last 6 months. Difficulties started after he was promoted to 7th Standard in school before which he had been an average student. His teachers complained of falling grades and diminished attention in class and soon he quit school. Gradually he seemed to be struggling with simple calculations and tasks required in day to day life and became clumsy. Hestarted wetting bed during sleep, often talked in a childish manner and indulged in smiling as well as crying for no apparent reason. He needed to be coaxed to clean himself after toilet, have his bath and change his clothes, which also required assistance of elders. It appeared to the parents that he was gradually becoming "dull" and child like.

Since last 8 months, as the aforesaid problems ramified, new symptoms appeared. Spontaneous movements and speech decreased, he walked with slight difficulty, and there were stiffness of his upper limbs and drooling. Since last 6 months, he became almost mute and indifferent to surroundings, spent most of his time lying or sitting idle and vacantly staring and smiling. On the other hand, he occasionally displayed unprovoked anger outbursts, characterised by screaming aloud and kicking movements of legs and he couldn't be pacified for a long time. These problems worsened over last few days.

There were no history of seizure, head injury, fever, substance abuse, coma, paralysis or loss of vision and no other involuntary or abnormal movements. No H/O any Neurological/ Psychiatric/ Hereditary illness in the family. He was second of six issues of his parents, was born of con-sanguinous marriage, and his birth and perinatal period, physical growth and developmental milestones were all within normal limits. Pre-morbidly he was an easy child.

Physical examination disclosed mild pallor, ataxia, stooping gait, drooling of saliva, mild hepatosplenomegaly without any signs of liver failure, dystonic posture, tremor and cogwheel rigidity of upper limbs and slightly increased tone of lower limbs. Mental status examination (MSE) revealed stooped posture, poor eye contact and fixed gaze, immobility, apathy, mutism, occasional grimacing and negativism. A score of 16 was obtained on BushFrancis Catatonia Rating (BFCR) scale. 
The routine laboratory investigations were found to be normal except for mild microcytic hypochromic anemia. Mild Hepato-splenomegaly were found on USG whole abdomen, and MRI Brain showed hyperintense signals in Basal Ganglia and Thalamic region. Ophthalmological opinion was sought for and K-F Rings were reported on Slit-Lamp examination. Thereafter neurological consultation was sought and a liaison was set up for his management. Serum Ceruloplasmin was found to be very low $(7 \mathrm{mg} / \mathrm{dl})$

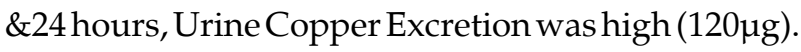
It was diagnosed to be a case of Catatonic Disorder due to Wilson's disease (293.89 by DSM-5).

Patient was admitted and parents were educated about his illness and management plans. Certain dietary restrictions were imposed. Pharmacotherapy was initiated comprising of Tab D-Penicillamine (starting with $125 \mathrm{mg} /$ day and increasing slowly over a week to $250 \mathrm{mg} /$ day) \& Tab Zinc Acetate (200mg/day) before food, Tab Pyridoxine $25 \mathrm{mg} /$ day, Tab Olanzapine $5 \mathrm{mg} /$ day and Tab Lorazapam $4 \mathrm{mg} /$ day. Over 2 weeks his agitation and grimacing decreased substantially and he was found to be more cooperative to the examiner. BFCR score reduced to 4 and he was discharged with same advice. However, his neurological symptoms persisted as before. On follow-up, 4 weeks later, his catatonic symptoms resolved almost completely and drooling, dystonia and tremor also improved significantly. Medications were continued except for reduction of lorazepam to $2 \mathrm{mg} /$ day and he is maintaining well with regular follow-up and compliance with medications.

\section{DISCUSSION}

Clinically evident liver disease may precedeneurologic manifestations in WD by as much as 10 years and mostpatients with neurologic symptoms have some degree of liverdisease at presentation. Presenting symptoms of liver diseasecan be highly variable, ranging from asymptomatic to acute hepatic failure and overt cirrhosis with all its complications ${ }^{[14]}$. No florid liver disease or abnormalities of liver enzymes were however found in the index patient apart from mild hepato-splenomegaly. Presence of agitation and behavioral disturbances led to his visit at Psychiatry OPD.

Half of patients with WD may undergo psychiatric hospitalization before WD is recognized. Psychiatric manifestations may precede neurological signs in the early stages of $\mathrm{WD}^{[6]}$. Catatonia on presentation has rarely been reported in WD. In India, two case reports described catatonia among other psychotic symptoms as presenting complains of WD, and the patients were satisfactorily managed by antipsychotics and Electroconvulsive therapy (ECT) [23,24]. The index case presented with catatonic symptoms and Bush-Francis catatonia rating gave a score of 16 .

The neurological abnormalities in WD can be classified as: (1) Akinetic-rigid syndrome similar to Parkinson's disease; (2) Pseudosclerosis dominated bytremor; (3) Ataxia; and (4) Dystonic syndrome. The characteristic tremor is a coarse, irregular proximal tremulousness with a "wing beating" appearance. Dystonia commonly involve the cranial region, and lead to dysarthria, or oropharyngeal dystonia. Facial grimacing, open jaw, speech changes, running saliva, etc are characteristic manifestations. A tremorrigidity syndrome ("juvenile Parkinsonism") should raise suspicion of Wilson's disease ${ }^{[25-27]}$. Presence of neurological symptoms like ataxia, tremor, rigidity and drooling in this boy led us to rule out any underlying organic cause of his behavioural and catatonic symptoms.Common differential diagnosis for young onset extrapyramidal syndrome (EPS) include Wilson's disease, young-onset Parkinson's disease, Hallervoden- Spartz disease, Huntington's disease, subacute sclerosing panencephalitis, hypoparathyroidism etc. WD was diagnosed in this case based on very low serum ceruloplasmin, high urinary copper excretion and K-F rings on slit-lamp examination.

On admission therapy was initiated with oral Penicillamine, Zinc Acetate, Pyridoxine, Olanzapine and Lorazapam with careful monitoring of extrapyramidal syndrome. His dramatic response within 2 weeks with marked resolution of agitation and 
other catatonic symptoms illustrates successful treatment with olanzapine and lorazepam without much side effects. The ataxia, dystonia and tremor that persisted during discharge after two weeks also substantially improved on follow-up after 4 weeks of discharge, possibly indicating effect of penicillamine and zinc on serum copper levels. Pyridoxine was added from beginning as a prophylactic measure due to pyridoxine antagonising properties of D-penicillamine.

To conclude, catatonia is a rare presenting symptom of Wilson's Disease and WD should be considered as a possibility in young patients presenting with catatonia, especially if co-morbid with neurological symptoms. Early diagnosis and prompt management of WD is associated with better overall outcome. Low dose of antipsychotics having low propensity for EPS can cautiously be used along with lorazepam in such cases for management of catatonic and psychotic symptoms.

\section{FOOTNOTES}

Source of support : Nil

Conflicts of interest : None

\section{REFERENCES}

[1] Tao TY, Gitlin JD. Hepatic copper metabolism : insights from genetic disease. Hepatology 2003; 37 : 1241-1247.

[2] Lutsenko S, Petris MJ. Function and regulation of the mammalian coppertransporting AT Pases : insights from biochemical and cell biological approaches. J Membr Biol 2003; 191 : 1-12.

[3] Scheinberg IH, Sternlieb I. Wilson's disease. In: Smith $\mathrm{Jr} \mathrm{LH}$, editor. Major problems in internal medicine, vol. 23. Philadelphia, PA: WB Saunders; 1984. p. 25-35.

[4] Gitlin JD. Wilson disease. Gastroenterology 2003; 125 : 1868-1877.

[5] Reilly M, Daly L, Hutchinson M. An epidemiological study of Wilson's disease in the Republic of Ireland. J Neurol Neurosurg Psychiatry 1993; 56 : 298-300.

[6] Akil M, Brewer GJ : Psychiatric and behavioral abnormalities in Wilson's disease. Adv Neurol 1995; 65 : 171-178.

[7] Dening TR, Berrios GE. Wilson's disease. Psychiatric symptoms in 195 cases. Arch Gen Psychiatry 1989; 46 : 112634.

[8] Akil M, Schwartz JA, Dutchak D, et al. The psychiatric presentations of Wilson's disease. J Neuropsychiatry Clin Neurosci $1991 ; 3: 377-82$.
[9] Brewer GJ, Yuzbasiyan-Gurkan V. Wilson disease. Medicine (Baltimore) 1992; 71 : 139-64.

[10] Seniow J, Mroziak B, Czlonkowska A, et al. Self-rated emotional functioning of patients with neurological or asymptomatic form of Wilson's disease. Clin Neuropsychol 2004; 17 : 367-373.

[11] Steindl P, Ferenci P, Dienes HP, et al. Wilson' s disease in patients presenting with liver disease : a diagnostic challenge. Gastroenterology 1997; 113 : 212-218.

[12] Gow PJ, Smallwood RA, Angus PW, et al. Diagnosis of Wilson's disease : an experience over three decades. Gut 2000; $46: 415-419$.

[13] Sanchez-Albisua I, Garde T, Hierro L et al. A high index of suspicion: the key to an early diagnosis of Wilson's disease in childhood. J Pediatr Gastroenterol Nutr 1999; 28 : 186190.

[14] EASL Clinical Practice Guidelines: Wilson's disease. Journal of Hepatology 2012 vol. 56 j 671-685.

[15] Dening TR, Berrios GE. Wilson's disease: a longitudinal study ofpsychiatric symptoms. Biol Psychiatry 1990; 28 : 255-65.

[16] Merle U, Schaefer M, Ferenci P, Stremmel W. Clinical Presentation, diagnosis and long-term outcome of Wilson disease - a cohort study. Gut 2007; 56 : $115-120$.

[17] Czlonkowska A, Tarnacka B, Litwin T, Gajda J, Rodo M. Wilson's disease - cause of mortality in 164 patients during 1992-2003 observation period. J Neurol 2005; 252 : 698-703.

[18] Stremmel W, Meyerrose KW, Niederau C, Hefter $H$, Kreuzpaintner G, Strohmeyer G. Wilson's disease : clinical presentation, treatment, and survival. Ann Intern Med 1991; $115: 720-726$.

[19] Lauterbach EC. Psychiatric management in Wilson's disease (progressive hepatolenticular degeneration). In: Lauterbach EC, editor. PsychiatricManagement in Neurological Diseases. American Psychiatric Press: Washington, DC; 2000. p. 93-137.

[20] Hoogenraad TU. Wilson's Disease. WB Saunders : London; 1996. p. 165.

[21] Negro Júnior PJ, Louzã Neto MR. Results of ECT for a case of depression in Wilson's disease (letter). J Neuropsychiatry Clin Neurosci 1995; 7 : 384.

[22] Shah N, Kumar D. Wilson's disease, psychosis and ECT. Convuls Ther 1997; $13: 278-9$.

[23] Sahoo MK, Avasthi A, Sahoo $M$ et al. Indian J Psychiatry 52(1), Jan-Mar 2010.

[24] Shetageri VN, Bhogale GS, Patil NM, etal. J Neuropsychiatry Clin Neurosci 24 : 3, Summer 2012.

[25] Brewer GJ. Neurologically presenting Wilson's Disease. CNS Drugs 2005; 19 : 185-192.

[26] LeWitt PA, Czlonkowska A. Wilson's disease. In : Lisak RP, Truong DD,Carroll WM, Bhidayasiri R, editors. International neurology, a clinical approach. Oxford UK: Wiley-Blackwell; 2009. p. 644-647.

[27] Svetel M, Potrebic A, Pekmezovic T, etal. Neuropsychiatric aspects of treated Wilson's disease. Parkinsonism Relat Disord 2009; 15 : 772-775. 